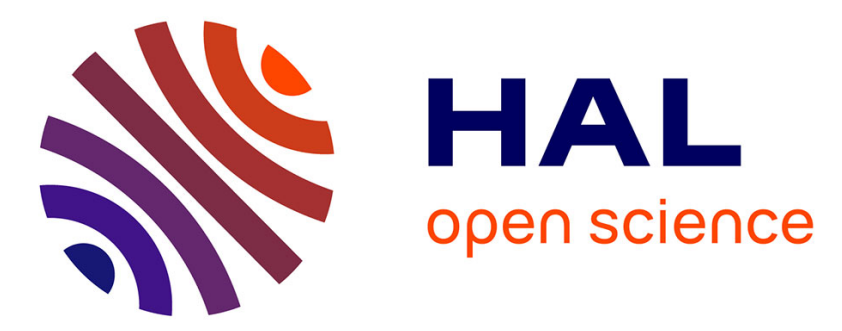

\title{
Analysis-suitable volume parameterization of multi-block computational domain in isogeometric applications
}

Gang Xu, Bernard Mourrain, Régis Duvigneau, André Galligo

\section{To cite this version:}

Gang $\mathrm{Xu}$, Bernard Mourrain, Régis Duvigneau, André Galligo. Analysis-suitable volume parameterization of multi-block computational domain in isogeometric applications. ComputerAided Design, 2013, Special Issue on Solid and Physical Modeling 2012, 45 (2), pp.395-404. 10.1016/j.cad.2012.10.022 . hal-00685002v2

\section{HAL Id: hal-00685002 \\ https://hal.inria.fr/hal-00685002v2}

Submitted on 19 Aug 2012

HAL is a multi-disciplinary open access archive for the deposit and dissemination of scientific research documents, whether they are published or not. The documents may come from teaching and research institutions in France or abroad, or from public or private research centers.
L'archive ouverte pluridisciplinaire HAL, est destinée au dépôt et à la diffusion de documents scientifiques de niveau recherche, publiés ou non, émanant des établissements d'enseignement et de recherche français ou étrangers, des laboratoires publics ou privés. 


\title{
Analysis-suitable volume parameterization of multi-block computational domain in isogeometric applications
}

\author{
Gang $\mathrm{Xu}^{\mathrm{a}}$ Bernard Mourrain ${ }^{\mathrm{b}}$ Régis Duvigneau ${ }^{\mathrm{b}}$ André Galligo ${ }^{\mathrm{c}}$ \\ a School of Computer Science and Technology, Hangzhou Dianzi University, Hangzhou 310018, P.R. China \\ b INRIA Sophia-Antipolis, 2004 Route des Lucioles, 06902 Cedex, France \\ ${ }^{\mathrm{c}}$ University of Nice Sophia-Antipolis, 06108 Nice Cedex 02, France
}

\begin{abstract}
Parameterization of computational domain is a key step in isogeometric analysis just as mesh generation is in finite element analysis. In this paper, we study the volume parameterization problem of multi-block computational domain in isogeometric version, i.e, how to generate analysis-suitable parameterization of the multi-block computational domain bounded by B-spline surfaces. Firstly, we show how to find good volume parameterization of single-block computational domain by solving a constraint optimization problem, in which the constraint condition is the injectivity sufficient conditions of B-spline volume parametrization, and the optimization term is the minimization of quadratic energy functions related to the first and second derivatives of B-spline volume parameterization. By using this method, the resulted volume parameterization has no self-intersections, and the isoparametric structure has good uniformity and orthogonality. Then we extend this method to the multi-block case, in which the continuity condition between the neighbor B-spline volume should be added to the constraint term. The effectiveness of the proposed method is illustrated by several examples based on three-dimensional heat conduction problem.
\end{abstract}

Key words: isogeometric analysis, volume parameterization, multi-block computational domain, heat conduction

\section{Introduction}

Isogeometric analysis (IGA for short) method proposed by Hughes et al. in [16] offers the possibility of bridging the gap between CAD and CAE. The approach uses the same spline representation both for the geometry and for the physical solutions, and thus avoids this costly forth and back transformations. This uniform framework provides more accurate and efficient ways to deal with complex shapes and to approximate the solutions of physical simulation problems. On the other hand, it also rises interesting geometric problems for analysis-suitable modeling tools 23 22, 24.

It is well known that mesh generation, which generates a discrete mesh of a computational domain from a given CAD object, is a key and the most time-consuming step in finite element analysis (FEA for short). It consumes about $80 \%$ of the overall design and analysis process 4 in automo-

Email addresses: xugangzju@gmail.com (Gang Xu), Bernard.Mourrain@inria.fr (Bernard Mourrain), Regis.Duvigneau@inria.fr (Régis Duvigneau), andre.galligo@unice.fr (André Galligo). tive, aerospace and ship industry. Parametrization of computational domain in IGA, which corresponds to the mesh generation in FEA, also has some impact on analysis result and efficiency. In particular, arbitrary refinements can be performed on the computational mesh in FEA, but in IGA if we compute with tensor product B-splines, we can only perform refinement operations in each parametric direction by knot insertion or degree elevation. Hence, parameterization of computational domain is also being important for IGA. As it is pointed by Cottrell et al. 6], one of the most significant challenges towards isogeometric analysis is constructing trivariate spline volume parameterizations from given CAD boundary representation.

From the viewpoint of graphics applications, volume parameterization of 3D models has been studied in [17/26 25]. As far as we know, there are only a few work on the parametrization of computational domains from the viewpoint of isogeometric applications. T. Martin et al. 18. proposed a method to fit a genus-0 triangular mesh by B-spline volume parameterization, based on discrete volumetric harmonic functions; this can be used to build computational domains for 3D IGA problems. E. Cohen et.al. [7] proposed the concept of analysis-aware 
modeling, in which the parameters of CAD models should be selected to facilitate isogeometric analysis. They also demonstrated the influence of parameterization of computational domains by several examples. J.M Escobar et al. proposed a method to construct a trivariate T-spline volume of complex genus-zero solids for isogeometric application [12. However, the proposed method demands a surface triangulation as input data. A variational approach for constructing NURBS parameterization of swept volumes is proposed by M. Aigner et al [1]. Given boundary CAD information, approximate implicitization technique is used for parametrization of $2 \mathrm{D}$ computational domain in 20]. In 27] 28, $r$-refinement method for generating optimal analysis-aware parameterization of computational domain is proposed. However, it only works for specified analysis problems. A general construction method for analysis-suitable planar B-spline parameterization in two-dimensional isogeometric problem is proposed in [29] based on harmonic mapping. In this paper, from the given boundary CAD information, the volume parameterization problem for multi-block computational domain is studied based on the trivariate generalization of the method proposed in 28 .

In IGA, the parameterization of a computational domain is determined by control points, knot vectors and the degrees of B-spline objects. For three-dimensional IGA problems, the knot vectors and the degree of the computational domain are determined by the given boundary surfaces. That is, given boundary surfaces, the quality of parameterization of computational domain is determined by the positions of inner control points. Hence, finding a good placement of the inner control points inside the computational domain, is a key issue. A basic requirement of the resulting volume parameterization for IGA is that it doesn't have self-intersections, so that it is an injective map from the parametrization domain to the computational domain. In order to get more accurate simulation results, the isoparametric structure in the computational domain should be as uniform as possible and have orthogonal isoparametric surfaces 2. In this paper, we study the volume parameterization problem of multi-block computational domain in isogeometric version, i.e, how to generate analysis-suitable parameterization of the multi-block computational domain bounded by B-spline surfaces. Our main contributions are:

- A constraint optimization framework is proposed to generate a multi-block volume parameterization without self-intersections, and the resulted isoparametric structure has good uniformity and orthogonality;

- Some classical results in the field of differential geometry related to parametric surfaces are generalized to the case of trivariate parametric volumes, such as the orthogonal conditions of isoparametric structure and the $C^{1}$ conditions between B-spline volumes.

- We test the volume parameterization results on heat conduction problem to show the effectiveness of the proposed method.
The remainder of the paper is organized as follows. Section 2 presents some preliminary on B-spline volume parameterization. Section 3 describes the constraint optimization method for single-block volume parameterization of 3D computational domain. Section 4 presents a volume parameterization framework for multi-block computational domain based on the proposed methods in Section 3 . Section 5 tests the volume parameterization results on heat conduction problem to show the effectiveness of the proposed method. Finally, we conclude this paper in Section 6 .

\section{Preliminary}

For a parameterization $\sigma$ from $\mathcal{P}:[a, b] \times[c, d] \times[e, f]$ to $\Omega \subset \mathbb{R}^{3}$, we define the boundary surfaces as the image of $\{a\} \times[c, d] \times[e, f],\{b\} \times[c, d] \times[e, f],\{c\} \times[a, b] \times[e, f],\{d\} \times$ $[a, b] \times[e, f],\{e\} \times[a, b] \times[c, d],\{f\} \times[a, b] \times[c, d]$ by $\sigma$. We say that $\sigma$ defines a regular boundary if these surfaces do not intersect pairwise, except at their boundary curves and if they have no self-intersection points.

We consider the following trivariate B-spline parameterization

$$
\begin{array}{r}
\sigma:(\xi, \eta, \zeta) \in \mathcal{P}:=[a, b] \times[c, d] \times[e, f] \mapsto \sigma(\xi, \eta, \zeta) \\
:=\sum_{\substack{0 \leq i \leq l \\
0 \leq j \leq m \\
0 \leq k \leq n}} \mathbf{c}_{i, j, k} N_{i}^{p}(\xi) N_{j}^{q}(\eta) N_{k}^{r}(\zeta),
\end{array}
$$

where $\mathbf{c}_{i, j, k} \in \mathbb{R}^{3}$ are the control points, $N_{i}^{p}(\xi), N_{j}^{q}(\eta)$ and $N_{k}^{r}(\zeta)$ are B-spline functions of degree $p, q$ and $r$ for a given knot vector on $[a, b],[c, d]$ and $[e, f]$. Note that in this paper we use knot vectors with multiple end knots to forces the volumes to interpolate the corner control points.

The derivative of $\sigma(\xi, \eta, \zeta)$ with respect to $\xi$ can be expressed in terms of the differences $\Delta_{i, j, k}^{1}:=$ $\left(\Delta_{i, j, k}^{1, x}, \Delta_{i, j, k}^{1, y}, \Delta_{i, j, k}^{1, z}\right)=\mathbf{c}_{i+1, j, k}-\mathbf{c}_{i, j, k}:$
$\quad \partial_{\xi} \sigma=\sum_{\substack{0 \leq i \leq l-1 \\ 0 \leq j \leq m \\ 0 \leq k \leq n}} \omega_{i, j, k}^{1} \Delta_{i, j, k}^{1} N_{i}^{p-1}(\xi) N_{j}^{q}(\eta) N_{k}^{r}(\zeta)$,

where $N_{i}^{p-1}(\xi)$ is the B-spline function with one degree less in $u, \omega_{i, j, k}^{1}$ is a positive factor.

Similarly, the derivative of $\sigma(\xi, \eta, \zeta)$ with respect to $\eta$ and $\zeta$ can be expressed as follows

$$
\begin{aligned}
& \partial_{\eta} \sigma=\sum_{\substack{0 \leq i \leq l \\
0 \leq j \leq m-1 \\
0 \leq k \leq n \\
0 \leq k}} \omega_{i, j, k}^{2} \Delta_{i, j, k}^{2} N_{i}^{p}(\xi) N_{j}^{q-1}(\eta) N_{k}^{r}(\zeta), \\
& \partial_{\zeta} \sigma=\sum_{\substack{0 \leq i \leq l \\
0 \leq j \leq m \\
0 \leq k \leq n-1}} \omega_{i, j, k}^{3} \Delta_{i, j, k}^{3} N_{i}^{p}(\xi) N_{j}^{q}(\eta) N_{k}^{r-1}(\zeta),
\end{aligned}
$$

where

$$
\begin{aligned}
& \Delta_{i, j, k}^{2}=\left(\Delta_{i, j, k}^{2, x}, \Delta_{i, j, k}^{2, y}, \Delta_{i, j, k}^{2, z}\right)=\mathbf{c}_{i, j+1, k}-\mathbf{c}_{i, j, k}, \\
& \Delta_{i, j, k}^{3}=\left(\Delta_{i, j, k}^{3, x}, \Delta_{i, j, k}^{3, y}, \Delta_{i, j, k}^{3, z}\right)=\mathbf{c}_{i, j, k+1}-\mathbf{c}_{i, j, k}, \\
& \text { and } \omega_{i, j, k}^{2}, \omega_{i, j, k}^{3} \text { are positive factors. }
\end{aligned}
$$




\subsection{Non-linear sufficient condition based on Jacobian computation}

From [28, if the Jacobian determinant $\boldsymbol{J}(\sigma(\xi, \eta, \zeta))$ of trivariate B-spline parameterization satisfies $\boldsymbol{J}(\sigma(\xi, \eta, \zeta))>$ 0 , then $\sigma(\xi, \eta, \zeta)$ has no self-intersections.

From (1), (2), (3) and the product properties of Bsplines [19], the Jacobian determinant $\boldsymbol{J}(\sigma(\xi, \eta, \zeta))$ of Bspline surface can be computed as follows:

$$
\begin{aligned}
& \boldsymbol{J}(\sigma(\xi, \eta, \zeta))=\left|\begin{array}{ccc}
\sigma_{\xi}^{x} & \sigma_{\eta}^{x} & \sigma_{\zeta}^{x} \\
\sigma_{\xi}^{y} & \sigma_{\eta}^{y} & \sigma_{\zeta}^{y} \\
\sigma_{\xi}^{z} & \sigma_{\eta}^{z} & \sigma_{\zeta}^{z}
\end{array}\right| \\
= & \sum_{\substack{0 \leq i \leq l-1 \\
0 \leq j \leq m \\
0 \leq k \leq n}} \sum_{\substack{0 \leq i^{\prime} \leq n \\
0 \leq k^{\prime} \leq n}} \sum_{\substack{0 \leq i^{\prime \prime} \leq n \\
0 \leq j^{\prime \prime} \leq m}}^{0 \leq k^{\prime \prime} \leq n-1} N_{i}^{p-1}(\xi) N_{j}^{q}(\eta) N_{k}^{r}(\zeta) \\
& N_{i^{\prime}}^{p}(\xi) N_{j^{\prime}}^{q-1}(\eta) N_{k^{\prime}}^{r}(\zeta) N_{i^{\prime \prime}}^{p}(\xi) N_{j^{\prime \prime}}^{q}(\eta) N_{k^{\prime \prime}}^{r-1}(\zeta) \\
& \left|\begin{array}{ccc}
\Delta_{i, j, k}^{1, x} \\
\omega_{i, j, k}^{1,} \omega_{i^{\prime}, j^{\prime}, k^{\prime}}^{2} \omega_{i^{\prime \prime}, j^{\prime \prime}, k^{\prime \prime}}^{3, x} \\
\Delta_{i^{\prime}, j^{\prime}, k^{\prime}}^{1, j} & \Delta_{i^{\prime}, j^{\prime}, k^{\prime}}^{2, y} & \Delta_{i^{\prime}, j^{\prime}, k^{\prime}}^{3, j} \\
\Delta_{i^{\prime \prime}, j^{\prime \prime}, k^{\prime \prime}}^{1, z} & \Delta_{i^{\prime \prime}, j^{\prime \prime}, k^{\prime \prime}}^{2, z} \Delta_{i^{\prime \prime}, j^{\prime \prime}, k^{\prime \prime}}^{3, z}
\end{array}\right| \\
= & \sum_{i=0}^{3 l-1} \sum_{j=0}^{3 m-1} \sum_{k=0}^{3 n-1} G_{i j k} N_{i}^{3 p-1}(\xi) N_{i}^{3 q-1}(\eta) N_{k}^{3 r-1}(\zeta)
\end{aligned}
$$

Hence, the Jacobian of trivariate B-spline parameterization can be represented in the form of trivariate B-spline volume with higher degrees. From the convex hull property of B-splines 13, we have the following theorem.

Theorem 2.1 If $G_{i j k}>0$ in (4), then $\mathbf{J}(\sigma(\xi, \eta, \zeta))>0$, that is, $\sigma(\xi, \eta, \zeta)$ has no self-intersections.

This is a non-linear sufficient condition with respect to the inner control points. We will use it as constraint term in the constraint optimization method for volume generation in Section 3 .

\subsection{Linear constraint for injectivity.}

In order to be self-contained, the linear sufficient condition proposed in 27] for injectivity of a B-spline volume parameterization is presented in this subsection.

We denote by $\mathcal{C}_{i}$ the convex cone of $\mathbb{R}^{i}$ generated by the half rays $\mathbb{R}_{+} \cdot \frac{\Delta_{i, j, k}^{i}}{\left\|\Delta_{i, j, k}^{i}\right\|}, i=1,2,3$. If this cone is generated by two opposite vectors, which are on a straight line, we define $\mathcal{C}_{i}(\mathbf{c})$ as any half-plane. We say that two cones $\mathcal{C}_{1}, \mathcal{C}_{2}$ are transverse if $\mathbb{R} \cdot \mathcal{C}_{1}$ and $\mathbb{R} \cdot \mathcal{C}_{2}$ intersect only at $\{0\}$. We say that three cones $\mathcal{C}_{1}, \mathcal{C}_{2}, \mathcal{C}_{3}$ are cotransverse if

- 0 is a vertex of the convex hull of $\mathcal{C}_{1}, \mathcal{C}_{2}, \mathcal{C}_{3}$;

- the convex hull of the cones $\mathcal{C}_{i}, \mathcal{C}_{j}$ and the cone $\mathcal{C}_{k}$ are transverse for all $\{i, j, k\}=\{1,2,3\}$.

Given six boundary surfaces described by the controls points $\mathbf{c}_{i, 0, k}, \mathbf{c}_{i, l_{2}, k}, \mathbf{c}_{0, j, k}, \mathbf{c}_{l_{1}, j, k}, \mathbf{c}_{i, j, 0}, \mathbf{c}_{i, j, l_{3}}$, with $0 \leq i \leq$ $l_{1}, 0 \leq j \leq l_{2}, 0 \leq k \leq l_{3}$, we define the boundary cone $\mathcal{C}_{1}^{0}$

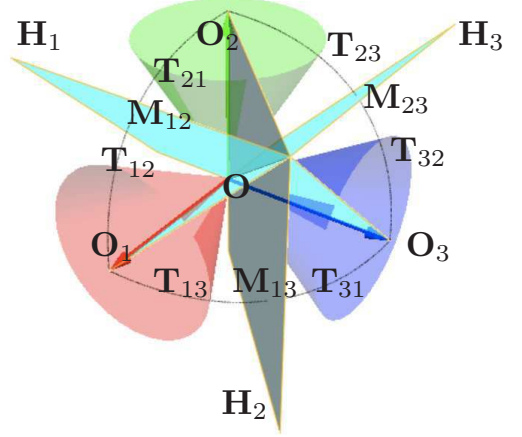

Fig.1. Construction of constraint plane.

(resp. $\left.\mathcal{C}_{2}^{0}, \mathcal{C}_{3}^{0}\right)$ as the cone generated by the vectors $\Delta_{i, j, 0}^{1}(\mathbf{c})$, $\Delta_{i, j, l_{3}}^{1}(\mathbf{c})$ for $0 \leq i \leq l_{1}-1,0 \leq j \leq l_{2}\left(\operatorname{resp} . \Delta_{0, j, k}^{2}(\mathbf{c})\right.$, $\Delta_{l_{2}, j, k}^{2}(\mathbf{c})$ for $0 \leq j \leq l_{2}-1,0 \leq k \leq l_{3}, \Delta_{i, 0, k}^{3}(\mathbf{c}), \Delta_{i, l_{2}, k}^{3}(\mathbf{c})$ for $\left.0 \leq i \leq l_{1}, 0 \leq k \leq l_{3}-1\right)$. We assume that these boundary surfaces form a regular boundary and that the three boundary cones $\mathcal{C}_{1}^{0}, \mathcal{C}_{2}^{0}, \mathcal{C}_{3}^{0}$ are cotransverse. Then we can find

- a plane $H_{0}$ such that $\forall \mathbf{u} \in \mathcal{C}_{i}^{0, *}(i=1,2,3), H_{0}(\mathbf{u})>0$, and

- a plane $H_{k}$ such that $\forall \mathbf{u} \in \mathcal{C}_{i}^{0, *} \cup \mathcal{C}_{j}^{0, *}, H_{k}(\mathbf{u})>0$ and $\forall \mathbf{u} \in \mathcal{C}_{k}^{0, *}, H_{k}(\mathbf{u})<0$ for $\{i, j, k\}=\{1,2,3\}$.

Such separating planes can be deduced easily from convex hull computations of finite sets of vectors, which are generating the cones $\mathcal{C}_{1}^{0}, \mathcal{C}_{2}^{0}, \mathcal{C}_{3}^{0}$ or their unions.

As shown in Fig 1] suppose that $\mathbf{O}$ is the origin, $\mathbf{O}_{1}$, $\mathbf{O}_{2}$ and $\mathbf{O}_{3}$ are the center of the circular cone $\mathcal{C}_{1}^{0}(\mathbf{c}), \mathcal{C}_{2}^{0}(\mathbf{c})$ and $\mathcal{C}_{3}^{0}(\mathbf{c})$, respectively. The great-circle arc connecting $\mathbf{O}_{1}$ and $\mathbf{O}_{2}$ intersects the circular cone $\mathcal{C}_{1}^{0}(\mathbf{c})$ and $\mathcal{C}_{2}^{0}(\mathbf{c})$ at $\mathbf{T}_{12}$ and $\mathbf{T}_{21}$. We can construct a orthogonal plane $\mathbf{H}_{1}$ of plane $\mathbf{O} \mathbf{T}_{12} \mathbf{T}_{21}$ through $\mathbf{O} \mathbf{M}_{12}$, where $\mathbf{M}_{12}$ is the middle points of $\mathbf{T}_{12} \mathbf{T}_{21}$. Let $F_{12}$ be the linear equation defining the plane $\mathbf{H}_{1}$. Then the vectors in $\mathcal{C}_{1}^{0}(\mathbf{c})$ and $\mathcal{C}_{2}^{0}(\mathbf{c})$ satisfy $F_{12} \leq 0$ and $F_{12} \geq 0$ respectively. Similarly, we can define $F_{13}$ and $F_{23}$ as the linear equations defining the plane $\mathbf{H}_{2}$ and $\mathbf{H}_{3}$.

Based on above the construction, the linear constraint conditions of inner control points for injective trivariate Bspline parameterization can be presented as follows,

$$
\left\{\begin{array}{l}
H_{0}\left(\mathbf{c}_{i+1, j, k}-\mathbf{c}_{i, j, k}\right)>0, H_{1}\left(\mathbf{c}_{i+1, j, k}-\mathbf{c}_{i, j, k}\right)<0, \\
H_{2}\left(\mathbf{c}_{i+1, j, k}-\mathbf{c}_{i, j, k}\right)>0, H_{3}\left(\mathbf{c}_{i+1, j, k}-\mathbf{c}_{i, j, k}\right)>0, \\
H_{0}\left(\mathbf{c}_{i, j+1, k}-\mathbf{c}_{i, j, k}\right)>0, H_{1}\left(\mathbf{c}_{i, j+1, k}-\mathbf{c}_{i, j, k}\right)>0, \\
H_{2}\left(\mathbf{c}_{i, j+1, k}-\mathbf{c}_{i, j, k}\right)<0, H_{3}\left(\mathbf{c}_{i, j+1, k}-\mathbf{c}_{i, j, k}\right)>0, \\
H_{0}\left(\mathbf{c}_{i, j, k+1}-\mathbf{c}_{i, j, k}\right)>0, H_{1}\left(\mathbf{c}_{i, j, k+1}-\mathbf{c}_{i, j, k}\right)>0, \\
H_{2}\left(\mathbf{c}_{i, j, k+1}-\mathbf{c}_{i, j, k}\right)>0, H_{3}\left(\mathbf{c}_{i, j, k+1}-\mathbf{c}_{i, j, k}\right)<0,
\end{array}\right.
$$

where $0<i<l_{1}, 0 \leq j<l_{2}, 0 \leq k<l_{3}$.

This set of conditions provides linear constraints for the injectivity of trivariate B-spline volume parameterization. In the following section, it will be used as constraint term in the quadratic programming method for the case 
when the boundary cones are cotransverse.

Remark 1. Note that the above two sufficient conditions only ensures the injectivity locally. The global injectivity can be guaranteed by the regularity of given boundary surfaces.

\section{Constraint optimization method for volume parametrization of computational domains}

In this section, we aim at finding injective volume parameterization of the computational domain with a uniform and orthogonal isoparametric net.

\subsection{Initial construction of inner control points}

In order to solve this constraint optimization problem, an initial construction of inner control points is required. We rely on the discrete Coons method to generate inner control points as initial value from boundary control points, which can be considered as the trivariate generalization of the method presented in [14].

Suppose that given boundary surfaces are Bspline surfaces, the opposite boundary B-spline surfaces have the same degree, number of control points and knot vectors. Given the boundary control points $\mathbf{c}_{0, j, k}, \mathbf{c}_{l, j, k}, \mathbf{c}_{i, 0, k}, \mathbf{c}_{i, m, k}, \mathbf{c}_{i, j, 0}, \mathbf{c}_{i, j, n}, \quad(i=0, \ldots, l, j=$ $0, \ldots, m, k=0, \ldots, n)$, then the interior control points $\boldsymbol{c}_{i, j, k}$ can be constructed as follows,

$$
\begin{aligned}
& \boldsymbol{c}_{i, j, k}=(1-i / l) \boldsymbol{c}_{0, j, k}+i / l \boldsymbol{c}_{l, j, k}+(1-j / m) \boldsymbol{c}_{i, 0, k} \\
& +j / m \boldsymbol{c}_{i, m, k}+(1-k / n) \boldsymbol{c}_{i, j, 0}+k / n \boldsymbol{c}_{i, j, n} \\
& -[1-i / l, i / l]\left[\begin{array}{cc}
\boldsymbol{c}_{0,0, k} & \boldsymbol{c}_{0, m, k} \\
\boldsymbol{c}_{l, 0, k} & \boldsymbol{c}_{l, m, k}
\end{array}\right]\left[\begin{array}{c}
1-j / m \\
j / m
\end{array}\right] \\
& -[1-j / m, j / m]\left[\begin{array}{rr}
\boldsymbol{c}_{i, 0,0} & \boldsymbol{c}_{i, 0, n} \\
\boldsymbol{c}_{i, m, 0} & \boldsymbol{c}_{i, m, n}
\end{array}\right]\left[\begin{array}{c}
1-k / n \\
k / n
\end{array}\right] \\
& -[1-k / n, k / n]\left[\begin{array}{rr}
\boldsymbol{c}_{0, j, 0} & \boldsymbol{c}_{l, j, 0} \\
\boldsymbol{c}_{0, j, n} & \boldsymbol{c}_{l, j, n}
\end{array}\right]\left[\begin{array}{c}
1-i / l \\
i / l
\end{array}\right] \\
& \left.+(1-k / n)[1-i / l, i / l]\left[\begin{array}{rr}
\boldsymbol{c}_{0,0,0} & \boldsymbol{c}_{0, m, 0} \\
\boldsymbol{c}_{l, 0,0} & \boldsymbol{c}_{l, m, 0}
\end{array}\right]\left[\begin{array}{r}
1-j / m \\
j / m
\end{array}\right]\right] \\
& \left.+k / n[1-i / l, i / l]\left[\begin{array}{rr}
\boldsymbol{c}_{0,0, n} & \boldsymbol{c}_{0, m, n} \\
\boldsymbol{c}_{l, 0, n} & \boldsymbol{c}_{l, m, n}
\end{array}\right]\left[\begin{array}{r}
1-j / m \\
j / m
\end{array}\right]\right]
\end{aligned}
$$

Then the corresponding B-spline volume has the following form

$$
\sigma(\xi, \eta, \zeta)=\sum_{i=0}^{l} \sum_{j=0}^{m} \sum_{k=0}^{n} \boldsymbol{c}_{i, j, k} N_{i}(\xi) N_{j}(\eta) N_{k}(\zeta) .
$$

where $N_{i}(\xi), N_{j}(\eta)$ and $N_{k}(\zeta)$ are B-spline function with knot vectors given by boundary surfaces.

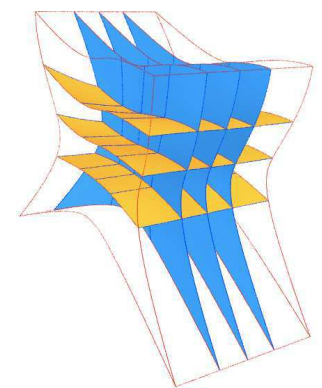

(a) Isoparametric surfaces and curves in a trivariate B-spline volume



(b) The partial derivatives at a point inside the volume

Fig.2. Isoparametric structure and partial derivatives in the trivariate $\mathrm{B}$-spline parameterization.

This initial construction of inner control points can be considered as an extension of boolean sum of ruled volumes and trilinear volumes constructed from give boundary surfaces as proposed in 11. The resulting inner control points lie in the convex hull of the boundary control points as the sum of the coefficients equals 1. For some given boundary surfaces, this construction may cause some self-intersections, and lead to an improper volume parameterization for IGA.

\subsection{Trivariate parametric volume with orthogonal and uniform grid}

An internal energy function of the computational domain will be used as an optimization term in a constraint optimization method to construct a computational domain with an uniform and orthogonal isoparametric grid.

In a trivariate B-spline parametrization, it has isoparametric surfaces and isoparametric curves with B-spline form as shown in Fig. 2(a). The partial derivatives at a point inside the trivariate volume are also shown in Fig. 2(b). For the trivariate parametric volume with orthogonal isoparametric surfaces, we have the following proposition.

Proposition 3.1 A trivariate parametric volume $\sigma(\xi, \eta, \zeta)$ has isoparametric grid with orthogonal isoparametric surfaces if and only if it satisfies the following condition

$$
\sigma_{\xi} \cdot \sigma_{\eta}=\sigma_{\xi} \cdot \sigma_{\zeta}=\sigma_{\eta} \cdot \sigma_{\zeta}=0
$$

The above proposition indicates that a trivariate parametric volume has orthogonal isoparametric surfaces if and only if each isoparametric surface has orthogonal isoparametric net. 
By minimizing the following energy functions, one can achieve isoparametric grid with orthogonal isoparametric surfaces,

$$
\iint\left|\sigma_{\xi} \cdot \sigma_{\eta}\right|+\left|\sigma_{\xi} \cdot \sigma_{\zeta}\right|+\left|\sigma_{\eta} \cdot \sigma_{\zeta}\right| d \xi d \eta d \zeta
$$

In view of the following inequalities,

$$
\begin{aligned}
\left|\sigma_{\xi} \cdot \sigma_{\eta}\right| & \leq \frac{\left\|\sigma_{\xi}\right\|^{2}+\left\|\sigma_{\eta}\right\|^{2}}{2} \\
\left|\sigma_{\xi} \cdot \sigma_{\zeta}\right| & \leq \frac{\left\|\sigma_{\xi}\right\|^{2}+\left\|\sigma_{\zeta}\right\|^{2}}{2} \\
\left|\sigma_{\eta} \cdot \sigma_{\zeta}\right| & \leq \frac{\left\|\sigma_{\eta}\right\|^{2}+\left\|\sigma_{\zeta}\right\|^{2}}{2}
\end{aligned}
$$

we replace (7) with

$$
\iiint\left\|\sigma_{\xi}\right\|^{2}+\left\|\sigma_{\eta}\right\|^{2}+\left\|\sigma_{\zeta}\right\|^{2} d \xi d \eta d \zeta
$$

The energy functions (8) can be seen as the trivariate generalization of stretch energy in [5]. The strain energy of parametric surface is related to the uniformity of the isoparameteric net as shown in [5]. Then we can generalize the strain energy in trivariate form as follows:

$$
\begin{aligned}
& \iiint\left(\left\|\sigma_{\xi \xi}\right\|^{2}+\left\|\sigma_{\eta \eta}\right\|^{2}+\left\|\sigma_{\zeta \zeta}\right\|^{2}\right. \\
& \left.+2\left\|\sigma_{\xi \eta}\right\|^{2}+2\left\|\sigma_{\xi \zeta}\right\|^{2}+2\left\|\sigma_{\eta \zeta}\right\|^{2}\right) d \xi d \eta d \zeta .
\end{aligned}
$$

In summary, combining (8) and (9), we use the following optimization term as objective function,

$$
\begin{aligned}
& \min \iiint\left(\left\|\sigma_{\xi}\right\|^{2}+\left\|\sigma_{\eta}\right\|^{2}+\left\|\sigma_{\zeta}\right\|^{2}\right) \\
& +\omega\left(\left\|\sigma_{\xi \xi}\right\|^{2}+\left\|\sigma_{\eta \eta}\right\|^{2}+\left\|\sigma_{\zeta \zeta}\right\|^{2}\right. \\
& \left.+2\left\|\sigma_{\xi \eta}\right\|^{2}+2\left\|\sigma_{\xi \zeta}\right\|^{2}+2\left\|\sigma_{\eta \zeta}\right\|^{2}\right) d \xi d \eta d \zeta .
\end{aligned}
$$

where $\omega$ is a positive constant.

\subsection{Non-linear constraint optimization method for volume parameterization}

After introducing the quadratic energy function in subsection 3.2, the following constraint optimization algorithm can be obtained based on the non-linear sufficient condition in subsection 2.1

Input: six boundary B-spline surfaces

Output: inner control points and the corresponding Bspline volume parameterization

- Construct the initial inner control points as in subsection 3.1

- Construct the constraint condition (4) from boundary B-spline surfaces as in Section 2.

- Solve the following constraint optimization problem by using sequential quadratic programming (SQP for short) method

$$
\begin{aligned}
& \min \iiint\left(\left\|\sigma_{\xi}\right\|^{2}+\left\|\sigma_{\eta}\right\|^{2}+\left\|\sigma_{\zeta}\right\|^{2}\right) \\
& +\omega\left(\left\|\sigma_{\xi \xi}\right\|^{2}+\left\|\sigma_{\eta \eta}\right\|^{2}+\left\|\sigma_{\zeta \zeta}\right\|^{2}\right. \\
& \left.+2\left\|\sigma_{\xi \eta}\right\|^{2}+2\left\|\sigma_{\xi \zeta}\right\|^{2}+2\left\|\sigma_{\eta \zeta}\right\|^{2}\right) d \xi d \eta d \zeta . \\
& \text { s.t. } \quad G_{i j k}>0
\end{aligned}
$$

- Generate the corresponding B-spline volume parameterization $\sigma(\xi, \eta, \zeta)$ as computational domain.

Remark 2. SQP is an iterative procedure which models the nonlinear optimization problem for a given iterate $x_{k}$ by a Quadratic Programming (QP for short) subproblem, solves that QP subproblem, and then uses the solution to construct a new iterate $x_{k+1}$. This construction is done in such a way that the sequence $x_{k}$ converges to the minimum of the optimization problem.

Remark 3. SQP optimization method may obtain a local minimum. To achieve the global minimum, initial position of inner control points is an important issue. From the examples we have tested in this paper, the initial construction of inner control points gives a good local minimum as final parameterization.

\subsection{Quadratic programming method for parametrization of computational domain}

If the boundary injectivity cones are transverse, we can propose a quadratic programming method for parameterization of computational domain as follows:

Input: six boundary B-spline surfaces

Output: inner control points and the corresponding Bspline volume parameterization

- Construct the initial inner control points as in subsection 3.1

- Construct the constraints condition (5) from boundary B-spline surfaces as in Section 2

- Solve the following constraint optimization problem by using a quadratic programming method,

$$
\begin{aligned}
& \min \iiint\left(\left\|\sigma_{\xi}\right\|^{2}+\left\|\sigma_{\eta}\right\|^{2}+\left\|\sigma_{\zeta}\right\|^{2}\right) \\
& +\omega\left(\left\|\sigma_{\xi \xi}\right\|^{2}+\left\|\sigma_{\eta \eta}\right\|^{2}+\left\|\sigma_{\zeta \zeta}\right\|^{2}\right. \\
& \left.+2\left\|\sigma_{\xi \eta}\right\|^{2}+2\left\|\sigma_{\xi \zeta}\right\|^{2}+2\left\|\sigma_{\eta \zeta}\right\|^{2}\right) d \xi d \eta d \zeta . \\
& \text { s.t. } \\
& \left\{\begin{array}{l}
H_{0}\left(\mathbf{c}_{i+1, j, k}-\mathbf{c}_{i, j, k}\right)>0, H_{1}\left(\mathbf{c}_{i+1, j, k}-\mathbf{c}_{i, j, k}\right)<0, \\
H_{2}\left(\mathbf{c}_{i+1, j, k}-\mathbf{c}_{i, j, k}\right)>0, H_{3}\left(\mathbf{c}_{i+1, j, k}-\mathbf{c}_{i, j, k}\right)>0, \\
H_{0}\left(\mathbf{c}_{i, j+1, k}-\mathbf{c}_{i, j, k}\right)>0, H_{1}\left(\mathbf{c}_{i, j+1, k}-\mathbf{c}_{i, j, k}\right)>0, \\
H_{2}\left(\mathbf{c}_{i, j+1, k}-\mathbf{c}_{i, j, k}\right)<0, H_{3}\left(\mathbf{c}_{i, j+1, k}-\mathbf{c}_{i, j, k}\right)>0, \\
H_{0}\left(\mathbf{c}_{i, j, k+1}-\mathbf{c}_{i, j, k}\right)>0, H_{1}\left(\mathbf{c}_{i, j, k+1}-\mathbf{c}_{i, j, k}\right)>0, \\
H_{2}\left(\mathbf{c}_{i, j, k+1}-\mathbf{c}_{i, j, k}\right)>0, H_{3}\left(\mathbf{c}_{i, j, k+1}-\mathbf{c}_{i, j, k}\right)<0,
\end{array}\right.
\end{aligned}
$$




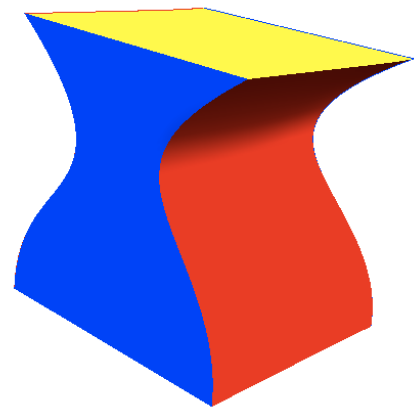

(a) boundary surfaces

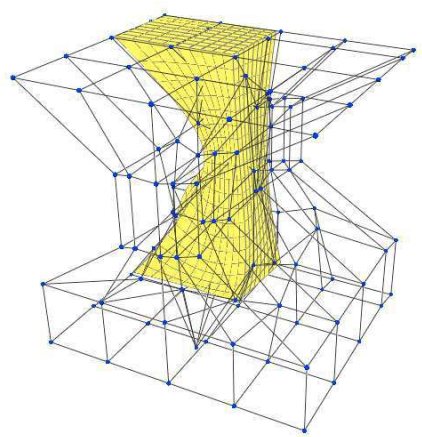

(d) optimized volume parameterization
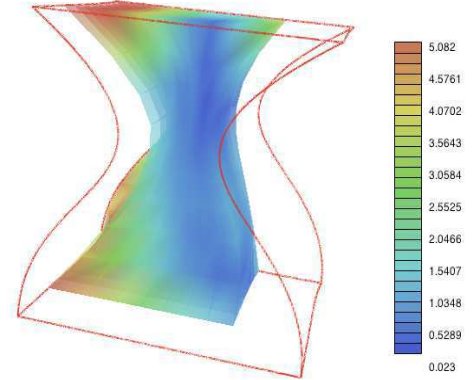

(g) orthogonality colormap in (c)



(b) boundary curves

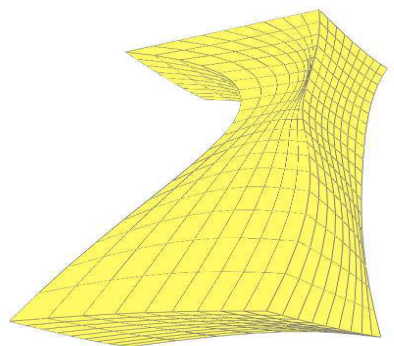

(e) parameterization details in (c)

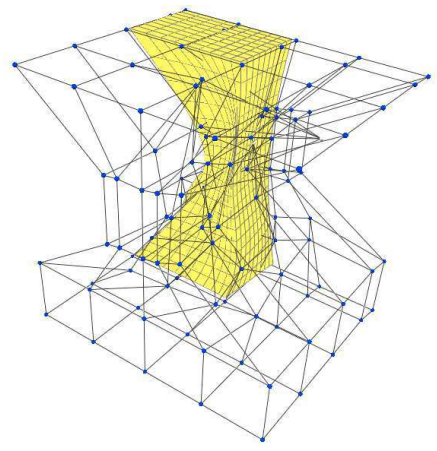

(c) Coons volume parameterization

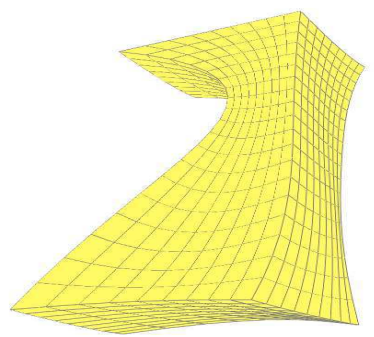

(f) parameterization details in $(d)$

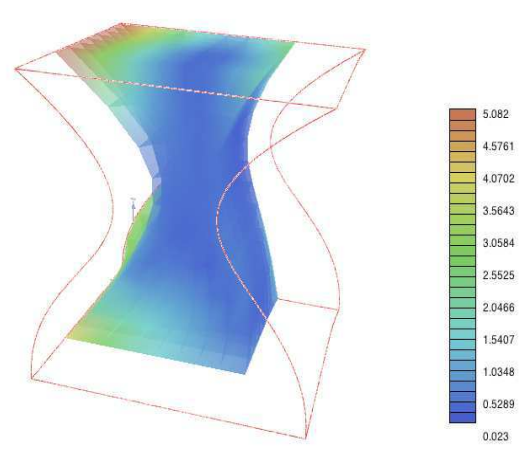

(h) orthogonality colormap in (d)

Fig.3. Volume parameterization example by non-linear constraint optimization method. The colormap in (h) has the same scale as (g).

- Generate the corresponding B-spline volume parameterization $\sigma(\xi, \eta, \zeta)$ as computational domain.

The first method is a non-linear constraint optimization algorithm, it can be used also for more general cases, including the case where the boundary injectivity cones are non-transverse. The second method is more efficient because the constraint conditions are linear and easy to compute. Fig. 3 shows a 3D example, which is drawn partly to illustrate the interior information of the volume. The given boundary B-spline surfaces and curves are shown in Fig. 3 (a) and 3 (b). Fig. 3 (c) presents the initial volume parametrization of computational domain constructed by discrete Coons method. There are some self-intersections on the initial parameterization. Fig. 3 (d) shows the final volume parameterization of computational domain without self-intersections constructed by the non-linear constraint optimization method. To illustrate the quality of the parameterization, the details of the B-spline volume parameterization are presented in Fig. 3 (e) and 3 (f). The optimization result in Figure 3 (f) avoids self-intersection and gives more uniform iso-parametric grid. We use the orthogonality colormap to show the orthogonality of volume parameterization on the isoparametric grids. In this paper, the orthogonality colormap is computed according to the value of $\frac{\cos \alpha+\cos \beta+\cos \gamma}{3}$, where $\alpha, \beta$ and $\gamma$ are angles formed by $\sigma_{\xi}, \sigma_{\eta}$ and $\sigma_{\zeta}$. From Fig 3 (g) and Fig $3(\mathrm{~h})$, optimized volume parameterization in Fig. 3 (d) has better orthogonality than Coons volume parameterization . The mean value of $\frac{\cos \alpha+\cos \beta+\cos \gamma}{3}$ in the optimized volume parameterization is also given in Table 2 . 


\section{Volume parameterization of multi-block computational domain}

In this section, we propose a volume parameterization framework for multi-block computational domain based on the proposed methods in Section 3 .

\section{1. $C^{1}$ condition of trivariate $B$-spline parametric volumes}

High continuity is one of the advantages in isogeometric analysis. For volume parameterization of multi-block computational domain, $C^{1}$ continuity between neighboring trivariate B-spline parametric volume is often required. Here we will propose the $C^{1}$ continuity conditions under which two B-spline parametric volume are differentiable.

Assume that the blocks are the same degree $(p, q, r)$ and $C^{0}$, that is, they share a common boundary surface. Additionally, define each B-spline block over an arbitrary domain, thus we have $\sigma_{1}(\xi, \eta, \zeta)$ defined over $\left[\xi_{0}, \xi_{1}\right] \times\left[\eta_{0}, \eta_{1}\right] \times$ $\left[\zeta_{0}, \zeta_{1}\right]$ and $\sigma_{2}(\xi, \eta, \zeta)$ defined over $\left[\xi_{1}, \xi_{2}\right] \times\left[\eta_{0}, \eta_{1}\right] \times\left[\zeta_{0}, \zeta_{1}\right]$. In order for $\sigma_{1}(\xi, \eta, \zeta)$ and $\sigma_{2}(\xi, \eta, \zeta)$ to be $C^{1}$ we require

$$
\left.\frac{\partial}{\partial \xi} \sigma_{1}(\xi, \eta, \zeta)\right|_{\xi=\xi_{1}}=\left.\frac{\partial}{\partial \xi} \sigma_{2}(\xi, \eta, \zeta)\right|_{\xi=\xi_{1}}
$$

Drawing from the control point interpretation of the partials in Eq.(1) and applying the chain rule, we have

$$
\sum_{\substack{0 \leq j \leq m \\ 0 \leq k \leq n}} \omega_{i, j, k}^{1,1} \Delta_{i, j, k}^{1,1} N_{j}^{q} N_{k}^{r}=\sum_{\substack{0 \leq j \leq m \\ 0 \leq k \leq n}} \omega_{i, j, k}^{1,2} \Delta_{i, j, k}^{1,2} N_{j}^{q} N_{k}^{r} .
$$

From the linear independence property of B-spline function, we have

$$
\omega_{i, j, k}^{1,1} \Delta_{i, j, k}^{1,1}=\omega_{i, j, k}^{1,2} \Delta_{i, j, k}^{1,2}, i=0, \ldots, l,
$$

where $\omega_{i, j, k}^{1,1}$ and $\omega_{i, j, k}^{1,2}$ are positive factors,

$$
\begin{aligned}
& \Delta_{i, j, k}^{1,1}=\mathbf{c}_{i+1, j, k}^{1}-\mathbf{c}_{i, j, k}^{1}, \\
& \Delta_{i, j, k}^{1,2}=\mathbf{c}_{i+1, j, k}^{2}-\mathbf{c}_{i, j, k}^{2},
\end{aligned}
$$

in which $\mathbf{c}_{i, j, k}^{1}$ and $\mathbf{c}_{i, j, k}^{2}$ are control points of trivariate Bspline volume $\sigma_{1}(\xi, \eta, \zeta)$ and $\sigma_{2}(\xi, \eta, \zeta)$ respectively.

From Eq.12), $C^{1}$ B-spline blocks satisfy the criteria that the three control points in each row of along their boundary surfaces are collinear, and the collinear points are positioned in the ratio dictated by the domain as shown in Fig. 4 .

\subsection{Multi-block volume parameterization with $C^{1}$ constraints}

After introducing the $C^{1}$ condition for B-spline parametric volumes, a framework for multi-block volume parameterization can be derived.

Input: multi-block computational domain with boundary spline surfaces

Output: inner control points and the corresponding

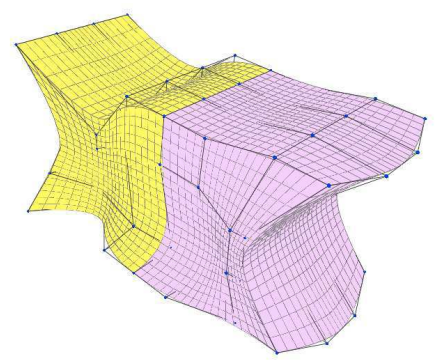

(a) $C^{1}$ B-spline blocks

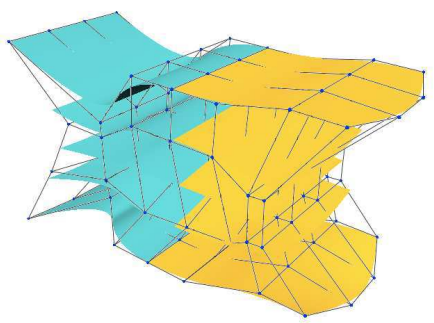

(b) Isoparametric surfaces and control lattices in $C^{1}$ B-spline blocks

Fig.4. $C^{1}$ condition of trivariate B-spline parametric volumes

multi-block B-spline volume parameterization with $C^{1}$ constraints

- Construct the initial inner control points for each block $\sigma^{i}$ as in subsection 3.1

- Construct the constraints condition (4) from boundary spline surfaces for each block $\sigma^{i}$ as in Section 2 .

- Solve the following constraint optimization problem to obtain the inner control points

$$
\begin{aligned}
& \min \sum_{\lambda=0}^{N} \iiint\left(\left\|\sigma_{\xi}^{\lambda}\right\|^{2}+\left\|\sigma_{\eta}^{\lambda}\right\|^{2}+\left\|\sigma^{\lambda} \zeta\right\|^{2}\right) \\
& +\omega\left(\left\|\sigma^{\lambda} \xi \xi\right\|^{2}+\left\|\sigma_{\eta \eta}^{\lambda}\right\|^{2}+\left\|\sigma_{\zeta \zeta}^{\lambda}\right\|^{2}\right. \\
& \left.+2\left\|\sigma_{\xi \eta}^{\lambda}\right\|^{2}+2\left\|\sigma_{\xi \zeta}^{\lambda}\right\|^{2}+2\left\|\sigma_{\eta \zeta}^{\lambda}\right\|^{2}\right) d \xi d \eta d \zeta . \\
& \text { s.t. }\left\{\begin{array}{l}
G_{i j k}^{\lambda}>0, \lambda=0, \ldots, N \\
\omega_{i, j, k}^{1, \lambda_{1}} \Delta_{i, j, k}^{1, \lambda_{1}}=\omega_{i, j, k}^{1, \lambda_{2}} \Delta_{i, j, k}^{1, \lambda_{2}}, i=0, \ldots, l,
\end{array}\right.
\end{aligned}
$$

- Generate the corresponding $C^{1}$ multi-block B-spline volume parameterization as computational domain.

Remark 4. The continuity between input neighboring boundary B-spline surfaces on different block should be at least $C^{1}$.

Remark 5. The quadratic programming method proposed in Section 3 can also be extended to the multi-block case in a similar way. Different from the single-block case, the control points to be determined on the common surface between two blocks should simultaneously satisfy the geometric conditions derived from these two blocks.

\section{Examples and comparison}

Starting from a trivariate B-spline volume as computational domain, a general framework of an isogeometric 


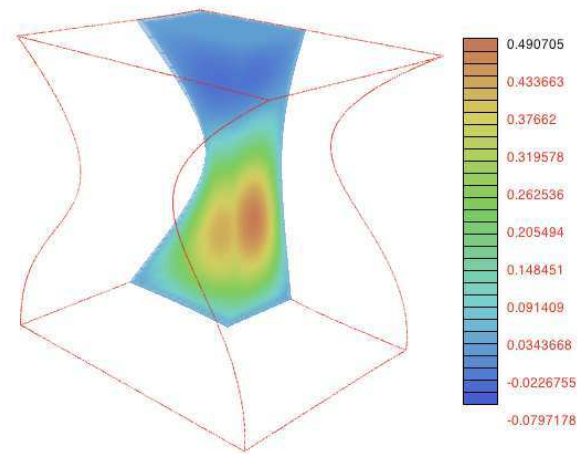

(a) solution field with respect to Coons parameterization


(c) error colormap with respect to Coons parameterization



(b) solution field with respect to optimized parameterization

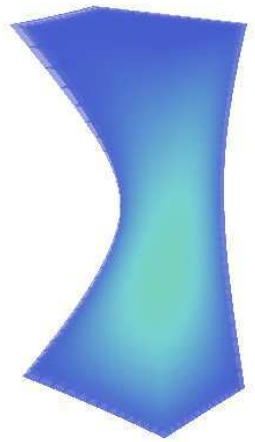

(d) error colormap with respect to optimized parameterization

Fig.5. Simulation example of non-linear constraint optimization method. The colormap in (d) has the same scale as (c).

solver for 3D heat conduction problem (13) has been implemented as a plugin in the AXEL 11 platform, yielding a B-spline volume as solution field. The proposed multi-block constraint optimization methods are implemented as a part of the isogeometric toolbox of the project EXCITING 2 .

In this paper, we test the different parameterizations of computational domains for the following heat conduction problem,

$$
\begin{aligned}
& \boldsymbol{\nabla}(\kappa(\mathbf{x}) \boldsymbol{\nabla} T(\mathbf{x}))=f(\mathbf{x}) \quad \text { in } \Omega \\
& T(\mathbf{x})=T_{0}(\mathbf{x}) \text { on } \partial \Omega_{D} \\
& \kappa(\mathbf{x}) \frac{\partial T}{\partial \mathbf{n}}(\mathbf{x})=\Phi_{0}(\mathbf{x}) \text { on } \partial \Omega_{N},
\end{aligned}
$$

where $\mathbf{x}$ are the Cartesian coordinates, $T$ represents the temperature field and $\kappa$ the thermal conductivity. Dirichlet and Neumann boundary conditions are applied on $\partial \Omega_{D}$ and $\partial \Omega_{N}$ respectively, $T_{0}$ and $\Phi_{0}$ being the imposed temperature and thermal flux (n unit vector normal to the boundary). $f$ is a user-defined source function. In this paper, the source function is defined as

$$
\boldsymbol{f}(x, y, z)=-\frac{\pi^{2}}{3} \sin \left(\frac{\pi x}{3}\right) \sin \left(\frac{\pi y}{3}\right) \sin \left(\frac{\pi z}{3}\right) .
$$

The boundary condition is specified as $\boldsymbol{T}_{0}(\boldsymbol{x})=0$ and $\Phi_{0}(\boldsymbol{x})=0$.

\footnotetext{
$\overline{1 \text { http://axel.inria.fr/ }}$

2 http://exciting-project.eu/
}

For problems with unknown exact solution $T$, suppose that $T_{h}$ is the approximation solution obtained by isogeometric method, then the discrete error $e=T-T_{h}$. Hence, a posteriori error assessment can be obtained by resolving the following problem,

$$
\begin{array}{cc}
\Delta e=-f+\Delta T_{h} & \text { in } \Omega \\
e=0 & \text { on } \partial \Omega_{D}
\end{array}
$$

The approximation error $e$ from (15) also has a Bspline form. In order to achieve more accurate results for above problem, some h-refinement operation should be performed. Then we can obtain a good approximation of error volume. Though it is much more expensive, we can use it as an error assessment method to show the effectiveness of the proposed construction method of computational domain.

Fig.5(a) and5(b) presents the corresponding solution field obtained from the volume parameterization in Fig. 3 (c) and Fig. 3 (d). The corresponding simulation error obtained from (15) are illustrated in Fig. 5 (c) and 5(d) with same scale. The final parameterization obtained by the nonlinear constraint optimization method can achieve better simulation results than the initial Coons parametrization, which illustrates that the optimized volume parameterization is analysis-suitable.

Fig. 6] presents a volume parameterization example with four blocks. The given boundary B-spline surfaces and 


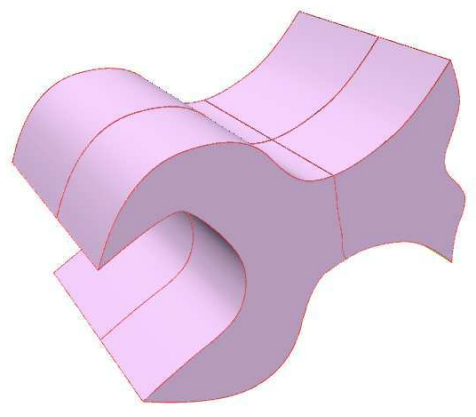

(a) boundary surfaces

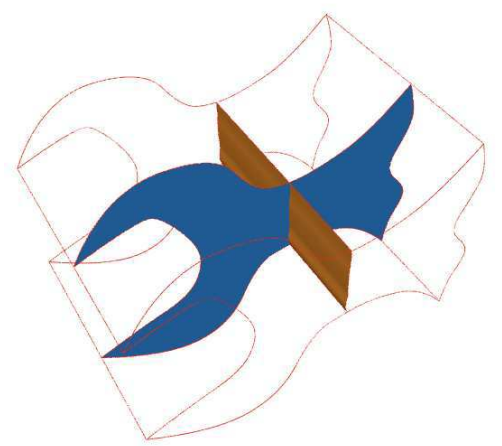

(b) boundary curves of blocks



(c) optimized volume parameterization



(d) solution field with respect to Coons parameterization

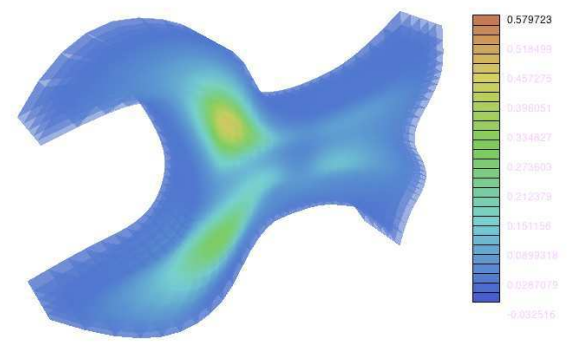

(f) error colormap with respect to Coons parameterization

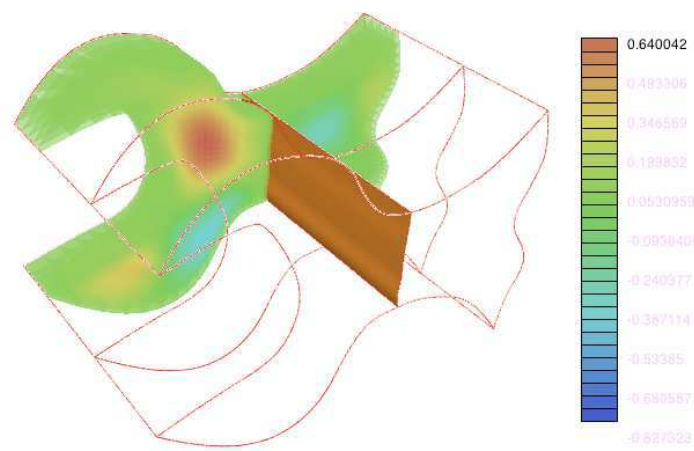

(e) solution field with respect to optimized parameterization

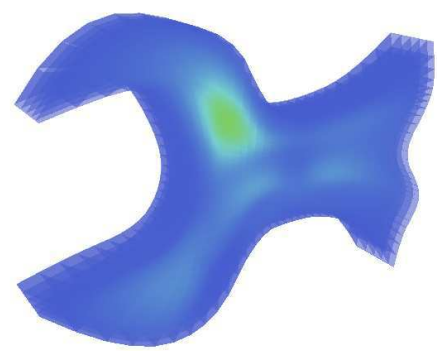

(g) error colormap with respect to optimized parameterization

Fig.6. Volume parameterization example of computational domain with four blocks. The colormap in (g) has the same scale as (f).

the block partition are shown in Fig.6 (a) and 6 (b). Fig. 7 (c) shows the optimized volume parameterization of computational domain without self-intersections constructed by the non-linear constraint optimization method. Fig. 6] (d) and 6] (e) shows the corresponding solution field obtained from Coons parameterization and optimized parameterization. The corresponding simulation error obtained from (15) are shown in Fig.6 (f) and 6 (g) with same scale. In order to show the effectiveness of the analysis-suitable volume parameterization, the global cross sectional view of the computational results and error colormap are illustrated. We can find that the optimized parameterization obtained by the non-linear constraint optimization method with $C^{1}$ continuity constraints can achieve better simula- tion results than the initial Coons parameterization. In order to show the $C^{1}$ continuity between two blocks, we compute the first derivatives at three sampling points for common boundary surfaces between $\sigma_{1}$ and $\sigma_{2}$ and the common boundary surface between $\sigma_{3}$ and $\sigma_{2}$, which are presented in Table 1

Fig. 7 shows a volume parameterization example with five blocks. The given boundary spline surfaces and curves are shown in Fig. 7(a) and 7(b). Fig. 7(c)(d) present the optimized volume parameterization of computational domain without self-intersections by the quadratic programming method. In order to illustrate the effectiveness of the proposed method, the global cross sectional view of the orthogonality colormap is shown in Fig 7 (e). 


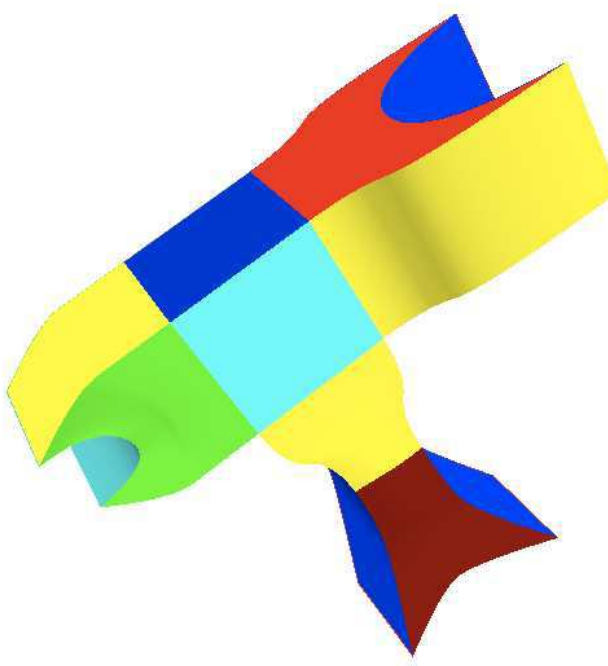

(a) boundary surfaces

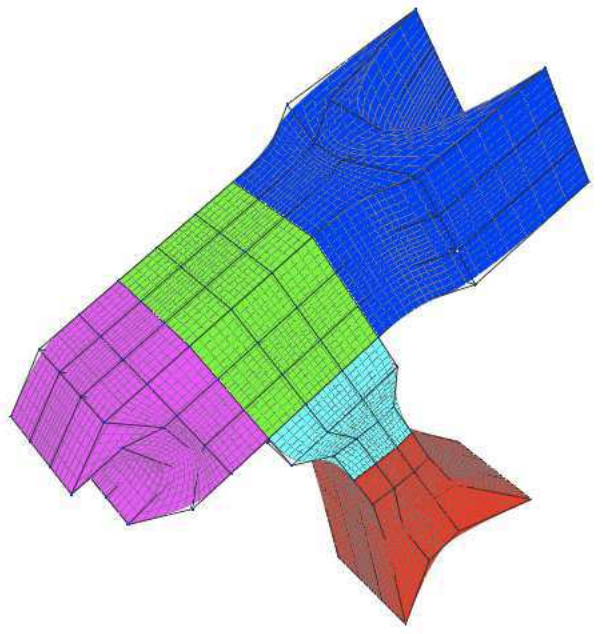

(c) optimized volume parameterization

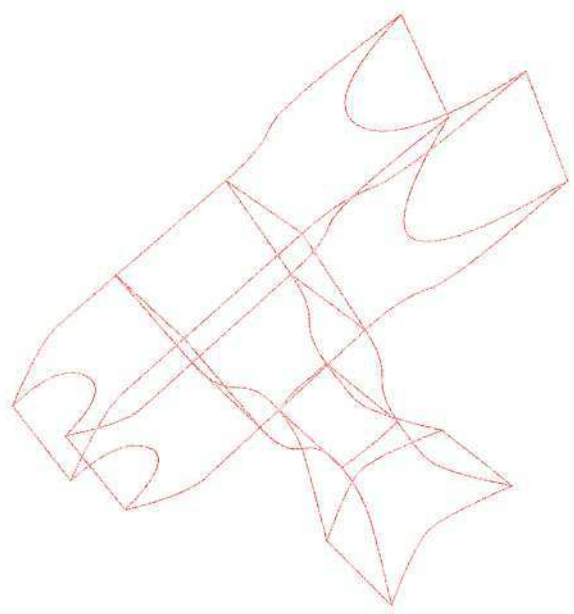

(b) boundary curves

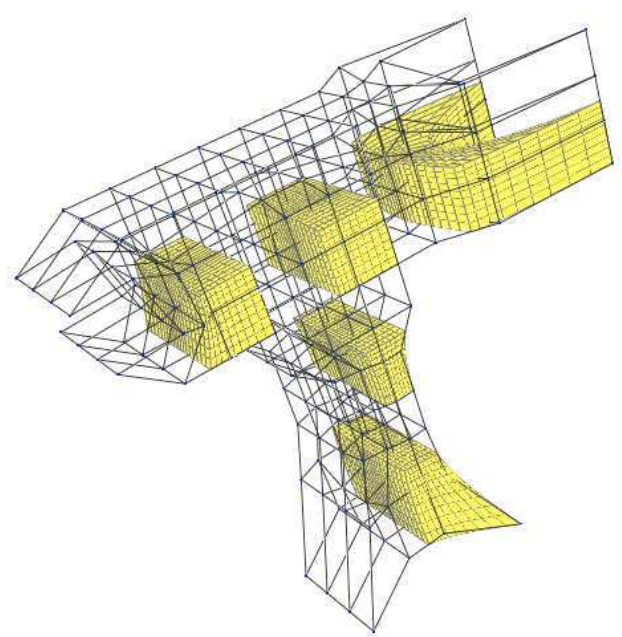

(d) interior view of optimized volume parameterization

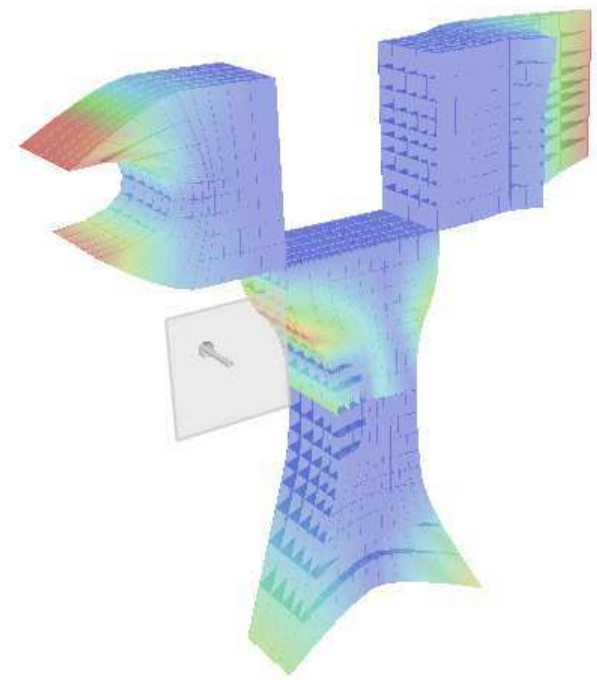

(e) interior view of orthogonality colormap

Fig.7. Volume parameterization example of computational domain with five blocks. 
Table 1

The first derivatives at sampling points on the common boundary surfaces in Fig. 6

\begin{tabular}{cc|cc}
\hline$\frac{\partial \sigma_{1}}{\partial \xi}$ & $\frac{\partial \sigma_{2}}{\partial \xi}$ & $\frac{\partial \sigma_{2}}{\partial \eta}$ & $\frac{\partial \sigma_{3}}{\partial \eta}$ \\
\hline$(0,0.16,1)$ & $(0,0.16,1)$ & $(-2.21,1.58,0)$ & $(-2.21,1.58,0)$ \\
$(0,0,1.33)$ & $(0,0,1.33)$ & $(0.73,1.03,0)$ & $(0.73,1.03,0)$ \\
$(0,0.35,2.98)$ & $(0,0.35,2.98)$ & $(1.07,3.04,0)$ & $(1.07,3.04,0)$ \\
\hline
\end{tabular}

Table 2

Quantitative data for volume parameterization in Fig 3 Fig 6 and Fig 7 \# deg.: degree of B-spline parameterization; \# con.: number of control points; KVI: knot vector information ; \# iter.: number of optimization iterations; \# CT.: computational time in seconds; \# MVA: mean value of orthogonality metric $\frac{\cos \alpha+\cos \beta+\cos \gamma}{3}$.

\begin{tabular}{|c|c|c|c|c|c|c|}
\hline Example & \# Deg. & \# Con. & KVI & \# Iter & \# CT. & \# MVA. \\
\hline Fig 3 & $p=q=r=3$ & 125 & {$[0,0,0,0,1,2,2,2,2]$} & 5 & 2.52 & 0.2033 \\
\hline Fig 6 & $p=q=r=3$ & 512 & {$[0,0,0,0,1,2,3,3,3,3]$} & 9 & 4.13 & 0.2567 \\
\hline Fig] & $p=q=r=2$ & 320 & {$[0,0,0,1,2,2,2]$} & 8 & 3.35 & 0.1356 \\
\hline
\end{tabular}

Quantitative data of volume parameterization method presented in Fig, 3. Fig, 6 and Fig[7 are summarized in Table 2

\section{Conclusion}

Analysis-suitable volume parameterization of computational domain plays an important role in isogeometric analysis as mesh generation in finite element analysis. In this paper, volume parameterization problem of multiblock computational domain in isogeometric applications is studied. This problem is solved in a constraint optimization framework, in which the constraint condition is the injectivity sufficient conditions of B-spline volume parametrization, and the optimization term is the minimization of quadratic energy functions related to the first and second derivatives of B-spline volume parameterization. The resulted volume parameterization has no selfintersections, and the isoparametric structure has good uniformity and orthogonality. Finally, the continuity condition between the neighbor B-spline volume are added into the constraint term to achieve a multi-block volume parameterization. Several examples are presented to show the effectiveness of the proposed method.

In the future, we will investigate the impact of boundary surfaces on the volume parameterization results. For a given CAD model, how to obtain an effective volume partition is also an interesting topic for isogeometric applications.

\section{Acknowledgements}

The authors wish to thank all anonymous referees for their valuable comments and suggestions. The authors are supported by the 7th Framework Program of the European Union, project SCP8-218536 "EXCITING". The first author is partially supported by the National Nature Science Foundation of China (No.61004117), the Zhejiang Provincial Natural Science Foundation of China (Nos.
Y1090718), the Defense Industrial Technology Development Program(A3920110002), the Scientific Starting Foundation of Hangzhou Dianzi University(No. KYS055611029) and the Open Project Program of the State Key Lab of CAD\&CG (A1105), Zhejiang University.

\section{References}

[1] M. Aigner, C. Heinrich, B. Jüttler, E. Pilgerstorfer, B. Simeon and A.-V. Vuong. Swept volume parametrization for isogeometric analysis. In E. Hancock and R. Martin (eds.), The Mathematics of Surfaces (MoS XIII 2009), LNCS vol. 5654(2009), Springer, 19-44.

[2] K.H. Brakhage and Ph. Lamby. Application of B-spline techniques to the modeling of airplane wings and numerical grid generation. Computer Aided Geometric Design, 25: 738-750, 2008.

[3] Y. Bazilevs, L. Beirao de Veiga, J.A. Cottrell, T.J.R. Hughes, and G. Sangalli. Isogeometric analysis: approximation, stability and error estimates for refined meshes. Mathematical Models and Methods in Applied Sciences, 6:1031-1090, 2006.

[4] Y. Bazilevs, V.M. Calo, J.A. Cottrell, J. Evans, T.J.R. Hughes, S. Lipton, M.A. Scott, and T.W. Sederberg. Isogeometric analysis using T-Splines. Computer Methods in Applied Mechanics and Engineering, 199(5-8): 229-263, 2010.

[5] G. Celniker and D. Gossard. Deformable curve and surface finite elements for free-form shape design. Computer Graphics (Procs of SIGGRAPH'91) 25(4): 257-266, 1991.

[6] Cottrell JA, Hughes TJR, Bazilevs Y (2009) Isogeometric Analysis: Toward Integration of CAD and FEA. John Wiley \& Sons, Chichester

[7] E. Cohen, T. Martin, R.M. Kirby, T. Lyche and R.F. Riesenfeld, Analysis-aware Modeling: Understanding Quality Considerations in Modeling for Isogeometric Analysis. Computer Methods in Applied Mechanics and Engineering, 199(5-8): 334$356,2010$.

[8] J.A. Cottrell, T.J.R. Hughes, and A. Reali. Studies of refinement and continuity in isogeometric analysis. Computer Methods in Applied Mechanics and Engineering, 196:4160-4183, 2007.

[9] M. Dörfel, B. Jüttler, and B. Simeon. Adaptive isogeometric analysis by local h-refinement with $\mathrm{T}$-splines. Computer Methods in Applied Mechanics and Engineering, 199(5-8): 264$275,2010$. 
[10] R. Duvigneau. An introduction to isogeometric analysis with application to thermal conduction. INRIA Research Report RR6957, June 2009

[11] G. Elber, Y. Kim, M. Kim. Volumetric boolean sum. Computer Aided Geometric Design, 29(7): 532-540, 2012.

[12] J.M. Escobara, J.M. Cascónb, E. Rodrígueza, R. Montenegro. A new approach to solid modeling with trivariate T-spline based on mesh optimization. Computer Methods in Applied Mechanics and Engineering, 200: 3210-3222, 2011.

[13] G. Farin. Curves and Surfaces for Computer Aided Geometric Design: A Practical Guide, 5th Edition. Morgan Kaufmann, San Mateo, CA, 2001.

[14] G. Farin and D. Hansford. Discrete coons patches. Computer Aided Geometric Design, 16(7):691-700, 1999.

[15] P. Gill, W. Murray, M Wright. Practical Optimization. Springer, 1981.

[16] T.J.R. Hughes, J.A. Cottrell, Y. Bazilevs. Isogeometric analysis: CAD, finite elements, NURBS, exact geometry, and mesh refinement. Computer Methods in Applied Mechanics and Engineering 194, 39-41: 4135-4195, 2005.

[17] Li X, Guo X, Wang H, He Y, Gu X, Qin H. Harmonic Volumetric Map- ping for Solid Modeling Applications. Proc. of ACM Solid and Physical Modeling Symposium 109-120, 2007, Association for Computing Machinery, Inc.

[18] T. Martin, E. Cohen, and R.M. Kirby. Volumetric parameterization and trivariate B-spline fitting using harmonic functions. Computer Aided Geometric Design, 26(6):648-664, 2009.

[19] K. M. Morken. Some identities for products and degree raising of splines. Constructive Approximation, 7: 195-208, 1991.

[20] T. Nguyen, B. Jüttler. Using approximate implicitization for domain parameterization in isogeometric analysis. International Conference on Curves and Surfaces, Avignon, France, 2010.

[21] N. Nguyen-Thanh, H. Nguyen-Xuan, S.P.A. Bordasd and T. Rabczuk. Isogeometric analysis using polynomial splines over hierarchical T-meshes for two-dimensional elastic solids. Computer Methods in Applied Mechanics and Engineering, 2011, 21-22: 1892-1908

[22] N. Nguyen-Thanh, J. Kiendl, H. Nguyen-Xuan, R. Wüchner, K.U. Bletzinger, Y. Bazilevs, T. Rabczuk. Rotation free isogeometric thin shell analysis using PHT-splines. Computer Methods in Applied Mechanics and Engineering, 2011, 47-48: 3410-3424

[23] M.A. Scott, X. Li, T.W. Sederberg, T.J.R. Hughes. Local refinement of analysis-suitable T-splines. Computer Methods in Applied Mechanics and Engineering, 2012, 213-216: 206-212

[24] P. Wang, J. Xu, J. Deng, F. Chen. Adaptive isogeometric analysis using rational PHT-splines. Computer-Aided Design, 2011, 43(11): 1438-1448

[25] J. Xia, Y. He, X. Yin, S. Han, and X. Gu. Direct product volume parameterization using harmonic fields. Proceedings of IEEE International Conference on Shape Modeling and Applications (SMI '10), 3-12, 2010.

[26] J. Xia, Y. He, S. Han, C.W. Fu, F. Luo, and X. Gu. Parameterization of star shaped volumes using Green's functions. Proceedings of Geometric Modeling and Processing (GMP '10), LNCS 6130, 219-235, 2010.

[27] G. Xu, B. Mourrain, R. Duvigneau, A. Galligo. Optimal analysis-aware parameterization of computational domain in $3 \mathrm{D}$ isogeometric analysis. Computer-Aided Design , 2012, to appear

[28] G. Xu, B. Mourrain, R. Duvigneau, A. Galligo. Parameterization of computational domain in isogeometric analysis: methods and comparison. Computer Methods in Applied Mechanics and Engineering, 2011, 200(23-24): 2021-2031

[29] G. Xu, B. Mourrain, R. Duvigneau, A. Galligo. Variational Harmonic Method for Parameterization of Computational Domain in 2D Isogeometric Analysis. Proceedings of 12th Conference on Computer-Aided Design and Computer Graphics, 223-228, Jinan, China, 2011 